\title{
THE EFFECTIVENESS OF LIFE-PRESERVING INVESTMENTS IN TIMES OF COVID-19
}

Jules H. van Binsbergen

Christian C. Opp

Working Paper 27382

http://www.nber.org/papers/w27382

\author{
NATIONAL BUREAU OF ECONOMIC RESEARCH \\ 1050 Massachusetts Avenue \\ Cambridge, MA 02138 \\ June 2020
}

We thank Jonathan Berk, Johannes van Binsbergen, and Meghan Opp for helpful comments and suggestions. All remaining errors are our own. The views expressed herein are those of the authors and do not necessarily reflect the views of the National Bureau of Economic Research.

NBER working papers are circulated for discussion and comment purposes. They have not been peer-reviewed or been subject to the review by the NBER Board of Directors that accompanies official NBER publications.

(C) 2020 by Jules H. van Binsbergen and Christian C. Opp. All rights reserved. Short sections of text, not to exceed two paragraphs, may be quoted without explicit permission provided that full credit, including $\odot$ notice, is given to the source. 
The Effectiveness of Life-Preserving Investments in Times of COVID-19

Jules H. van Binsbergen and Christian C. Opp

NBER Working Paper No. 27382

June 2020

JEL No. G31,H51,I1,I18

\section{ABSTRACT}

We analyze the effectiveness of preventive investments aimed at increasing agents' life expectancy, with a focus on influenza and COVID-19 mitigation. Maximizing overall life expectancy requires allocating resources across hazards so as to equalize investments' marginal effectiveness. Based on estimates for the marginal effectiveness of influenza vaccines, we determine the level of COVID-19 mitigation investments that would imply such equalization. Given current projections for COVID-19 mitigation costs, our results suggest that wide-spread influenza vaccination would be an effective life-preserving investment.

Jules H. van Binsbergen

The Wharton School

University of Pennsylvania

3620 Locust Walk

Philadelphia, PA 19104

and NBER

julesv@wharton.upenn.edu

Christian C. Opp

Simon Business School

University of Rochester

3-110N Carol Simon Hall

Rochester, NY 14627

and NBER

opp@rochester.edu 


\section{Introduction}

The COVID-19 pandemic has affected the United States in dramatic ways. As of May 11, more than 80,000 deaths have been linked to reported cases. To slow the spread of the virus, the government has imposed unprecedented restrictions, an economy-wide lockdown. As a result of mitigation responses, the economy has entered a historic recession. A record-high number of unemployment claims - representing $25 \%$ of American workers - were filed over the course of seven weeks since mid-March 2020. The April unemployment rate rose to a record 14.7\%, exceeding the previous record rate of $10.8 \%$ for data going back to 1948. GDP decreased $4.8 \%$ in the first quarter, and is projected to decline by $5.9 \%$ in 2020.1

While mitigation has severe consequences, it is motivated by two benefits associated with the slowing of the pandemic, also referred to as the "flattening of the curve." First, it provides time for the health care sector to develop treatments and vaccines. Whereas a large fraction of a population are expected to become infected absent mitigation, a lower fraction could end up getting infected if a vaccine became available during the time won by slowing the evolution of the pandemic. Second, mitigation lowers peak demand for medical treatments from hospitals, thereby ensuring that fatality rates do not increase due to capacity shortages. In light of these benefits, mitigation of some form is widely supported, although there is a debate on its optimal scale and nature.

In this paper, we shed light on this debate by analyzing the relative effectiveness of society's investments aimed at increasing agents' life expectancy, with a particular focus on influenza and COVID-19 mitigation. Individuals face a large variety of life hazards that can be reduced through various forms of investments, including vaccination, research, health care coverage, and behavioral changes. Yet, scarcity of resources implies that society always faces trade offs. Even when maximizing expected life expectancy is the only policy objective, doing everything possible to mitigate any one particular hazard is generally suboptimal. Due to non-linear returns to scale, such narrowly focused investments would generally cause large excess mortality relative to an allocation that spreads resources across areas and equalizes their marginal effectiveness. Based on estimates for the marginal effectiveness of influenza vaccines, we determine the level of COVID-19 mitigation investments that would imply such equalization. Given current projections for COVID-19 mitigation costs, our results suggest that wide-spread influenza vaccination would be a highly effective life-preserving investment.

In practice, investments' marginal effectiveness may differ across hazards for several rea-

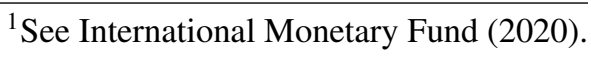


sons. At least five classes of explanations appear possibly relevant. First, in a decentralized market economy allocations across agents are largely dictated by wealth differences. Thus, any hazard that disproportionately affects wealthy agents will attract a relatively higher mitigation investment. Rich agents may already have minimized a range of hazards, given the abundance of available resources at their disposal, leading them to allocate larger amounts to other hazards that for poorer people are less important, in relative terms. While this class of explanations is generally likely important, it is not clear that it is a first-order determinant of differences in mitigation investments' effectiveness between COVID-19 and influenza, as the seasonal flu is still a relevant hazard for wealthier individuals.

Second, agents may have preferences that feature differing aversions to various causes of death. Yet, within a narrowly defined hazard category of viruses causing respiratory tract infections, this channel is unlikely to create much dispersion in mitigation investments' effectiveness.

Third, agents may be averse to fluctuations and risks associated with death rates..$^{2}$ Without mitigation, leading models predict a sharp rise in mortality rates absent COVID-19 mitigation. Yet, apart from this sharp rise, there is significant uncertainty about its magnitude and about the economic costs of mitigation activities. A related issue is that it is difficult for policy makers to fine-tune COVID-19 mitigation policies, since rules have to be simple and involve large groups of agents or businesses at a time, which implies discreteness in the adjustments of measures. As a result, policy makers have to worry about overshooting or undershooting. Risk aversion with respect to the size of the death toll would increase the willingness to pay for mitigation. On the other hand, uncertainty about the costs associated with mitigation activities would generally also command a risk premium, lowering the willingness to decide on far-reaching mitigation measures. The risk premia associated with benefits and costs of COVID-19 mitigation thus likely have partially off-setting effects. In contrast, in the case of influenza, the costs of vaccines are very predictable, but the fluctuations in benefits are quite substantial. The seasonal flu already causes significant with-in year and across-year fluctuations, although these fluctuations have attracted little attention in public discussions and policy proposals.

Fourth, agents may be subject to behavioral biases that neglect smaller risks and overweight salient hazards. Seeing pictures on television and on social media may lead to a subjective overrepresentation of risks associated with COVID-19. Conversely, the lack of coverage of other relevant hazards may lead to an underestimation of those risks. Biased beliefs are also likely amplified by a lack of accurate information and education. For instance, agents may confuse

\footnotetext{
${ }^{2}$ This point is distinct from the fact that COVID-19 fatality rates are likely to rise when hospital capacity limits are reached. This latter issue is already accounted for in the estimates we compute.
} 
case fatality rates with infection fatality rates for COVID-19. Whereas case fatality rates are widely reported, they are often more than 30 times higher than infection fatality rates, the latter of which is the relevant measure for agents' risk evaluations. For example, in France, the case fatality rate is $15 \%$, whereas the infection fatality rate is estimated to be $0.53 \%{ }^{3}$ Resulting biases in expectations could therefore quantitatively account for large dispersion in investments' effectiveness.

Fifth, institutional frictions may interact with behavioral biases. Politicians with preferences for staying in office are particularly averse to the possibility of being associated with salient negative outcomes. Thus, allocations that tilt investments toward such salient hazards may even be promoted by politicians with superior information.

In sum, behavioral and institutional distortions could plausibly play an important role in creating substantial dispersion in investments' effectiveness, although preference-based explanations and wealth dispersion could also be relevant factors. Irrespective of these channels, our analysis suggest that wide-spread influenza vaccination would be a highly effective lifepreserving investment.

\section{Data}

In this section, we describe the main data inputs and sources that we use in our computations.

\subsection{Demographics and Life Expectancy}

For the population distribution, we collect data from the Census, which reports the number of individuals by age. The usual range (or bucket) size for a reported age group is 5 or 10 years. We use the latest reported data from 2017. To arrive at an estimated population number $N_{i}$ for each age year $(i=1, \ldots, 100)$, we fit a spline through the reported data points. The results are plotted in I which reports for each age the estimated number of people in the U.S. population that have that age (in thousands) in 2017.

Next, we use data on life expectancy from the 2019 National Vital Statistics Report of the CDC. $\unlhd^{4}$ This report refers to data from 2017 and reports for each age $i$ what the life expectancy

\footnotetext{
${ }^{3}$ See Salje et al. (2020).

${ }^{4}$ See Centers for Disease Control and Prevention (2019b).
} 


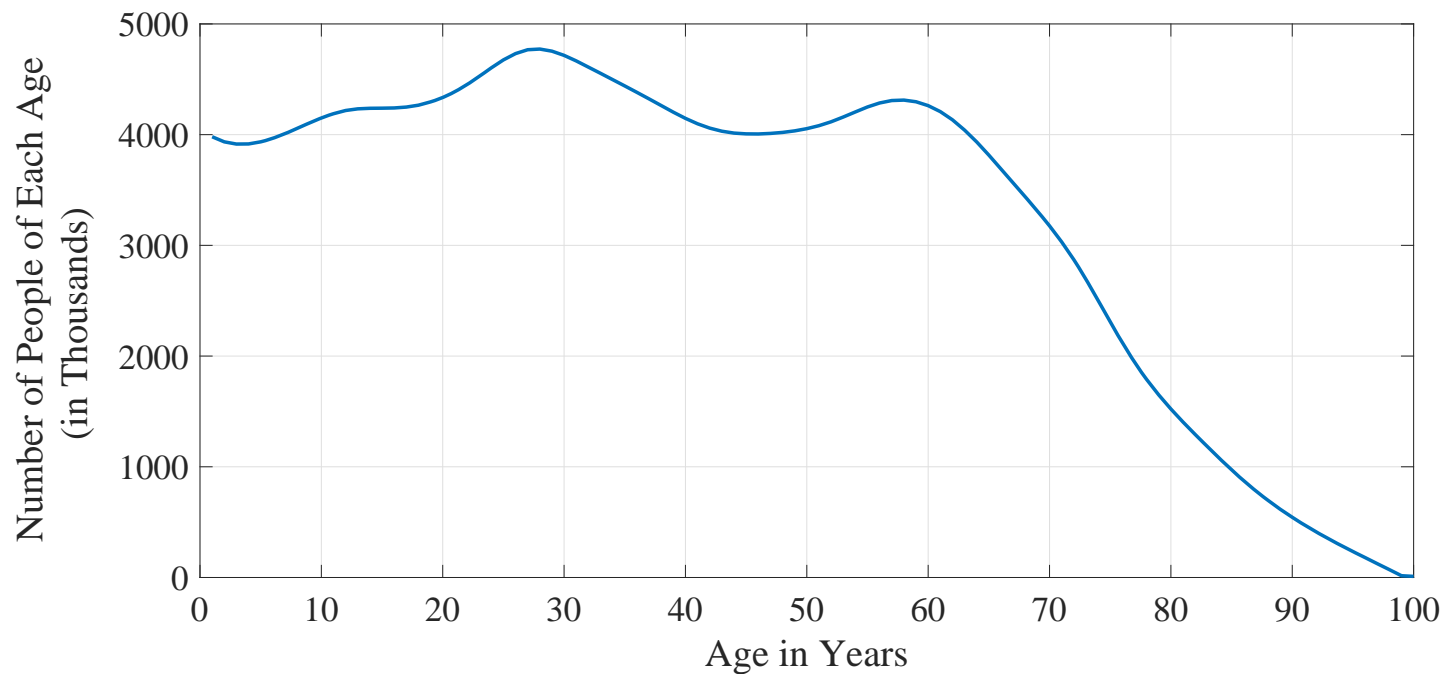

FIGURE I

Population by Age. The graph plots the distribution of the 2017 U.S. population by age (in thousands). The total population of the U.S. in that year was 326 million people.

$L_{i}$ at that age is. Figure $\Pi$ illustrates this data. The graph shows that a person born in the U.S. in 2017 had an average life expectancy just short of 80 years, whereas a person 85 years of age in 2017 had an average life expectancy of 6.5 years.

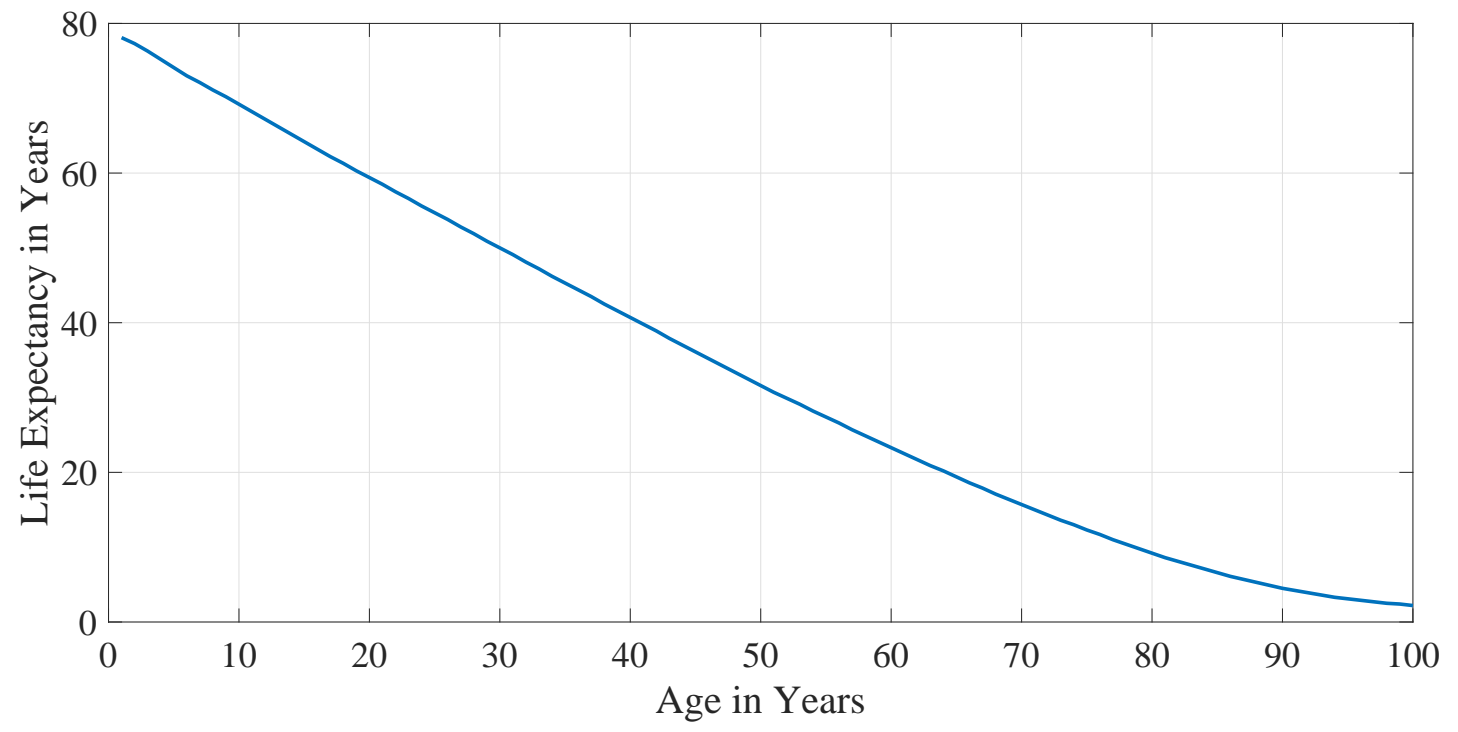

FIGURE II

Life Expectancy. The graph plots the life expectancy in 2017 for the U.S. population by age. 


\subsection{Number of Deaths by Cause and Age}

We also collect data on the most common causes of death in the United States in 2017, as reported by the $\mathrm{CDC} 5_{5}^{5}$ In Figure III, we report the total number of deaths by major cause. In total, just short of 3 million people died in the U.S. in 2017. The most common causes of death were heart disease at 647,457, malignant neoplasms (cancer) at 599,108 and accidents (including but not restricted to traffic) at 169,936. Traffic accidents in 2017 amounted to 40,231 deaths. The number of flu and pneumonia deaths in 2017 was 55,672. The number of estimated deaths for the 2017-2018 flu season (which includes data from 2018) is about 61,000.

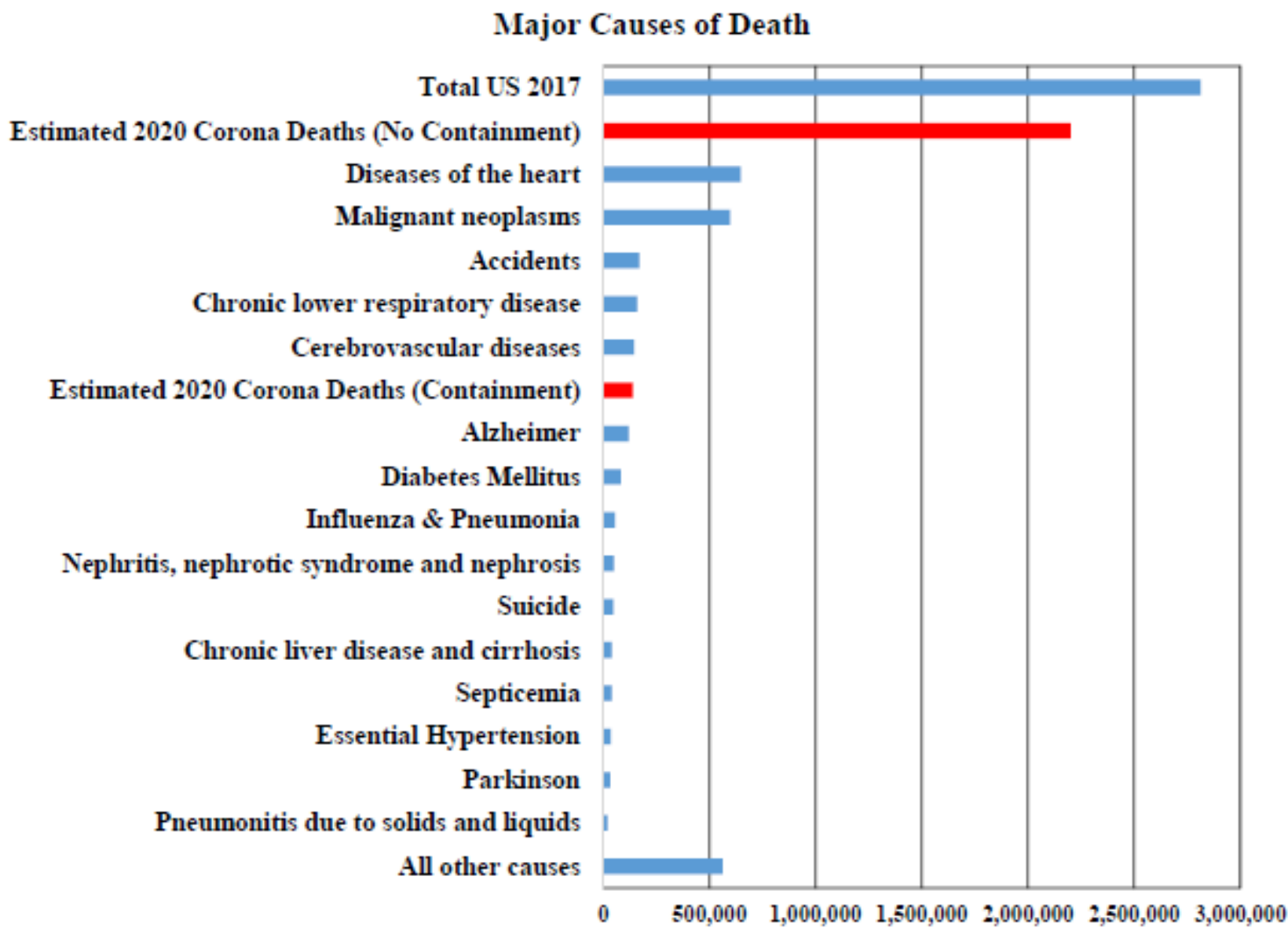

FIGURE III

Major Causes of Death. The bar graph shows the major causes of death and the number of associated deaths in 2017. As a comparison, we also plot (in red bars) the current number of estimated corona deaths under containment, as well as without containment (from Ferguson et al. 2020).

\footnotetext{
${ }^{5}$ See Centers for Disease Control and Prevention (2019b).
} 


\section{Life Years Lost}

In the next sections, we will compute for a range of diseases the number of life years lost due to that disease. Let $r_{i}^{k}$ denote the death rate of disease $k$ at age $\left.i(i=1, \ldots, 100)\right)$ per 100,000 people. The total number of deaths from disease $k$ at age $i$ is then given by:

$$
D_{i}^{k}=\frac{r_{i}^{k}}{100,000} N_{i}
$$

The life years lost for age group $i$ is then given by:

$$
\Theta_{i}^{k} \equiv D_{i}^{k} \times L_{i}
$$

and the total number of life years lost from disease $k$ across all age groups is given by:

$$
\Theta^{k} \equiv \sum_{i=1}^{100} \Theta_{i}^{k}
$$

\section{Influenza}

In this section, we analyze influenza and pneumonia related deaths and life years lost. We further collect data on current vaccination rates and flu vaccine effectiveness, and use these statistics to compute counterfactual flu incidence and death rates. Influenza is a particularly relevant cause of death for our analysis for several reasons. First, as the WHO states, "COVID-19 and influenza viruses have a similar disease presentation. That is, they both cause respiratory disease, which presents as a wide range of illness from asymptomatic or mild through to severe disease and death. ... both viruses are transmitted by contact, droplets and fomites. As a result, the same public health measures, such as hand hygiene and good respiratory etiquette (coughing into your elbow or into a tissue and immediately disposing of the tissue), are important actions all can take to prevent infection.' 6 Second, available data provide fairly precise predictions regarding the typical magnitudes of influenza related deaths. Third, existing studies yield information on the effectiveness of mitigation interventions, in particular, the effectiveness of influenza vaccines that are readily available at low costs.

\footnotetext{
${ }^{6}$ See World Health Organization 2020).
} 


\subsection{Death Rates and Life Years Lost}

The 2017-2018 flu season was relatively severe, with an estimated number of deaths over 60,000 . During the year 2017, the total number of deaths was 55,672. For each calendar year, the CDC reports death rates by age cohort, with buckets ranging from 1 year for babies to 4 years for small children, and 10 years for adults. We fit a spline through these data points to estimate for each age what the death rate from the flu is. The resulting estimates for $r_{i}^{\text {flu }}$ are plotted in Figure IV] It is important to stress that these death rates are not the probability of death conditional on having the flu. Rather, they represent the unconditional probability of dying from the flu in a given year by age.

Figure IV shows that the death rate is strongly increasing with age, much like the estimated rates for COVID-19 (see Figure XIII). As suggested by the fact that general life expectancy declines with age (Figure $\Pi$ ), older individuals are naturally more vulnerable to almost all causes of death - that is, their vulnerability is not specific to COVID-19 or influenza. In fact, influenza and COVID-19 are on par with the overall death rate in the population across age groups. In contrast, car accidents and cancer tend to affect younger people more than average, and Alzheimer's disease tends to affect older people much more.7

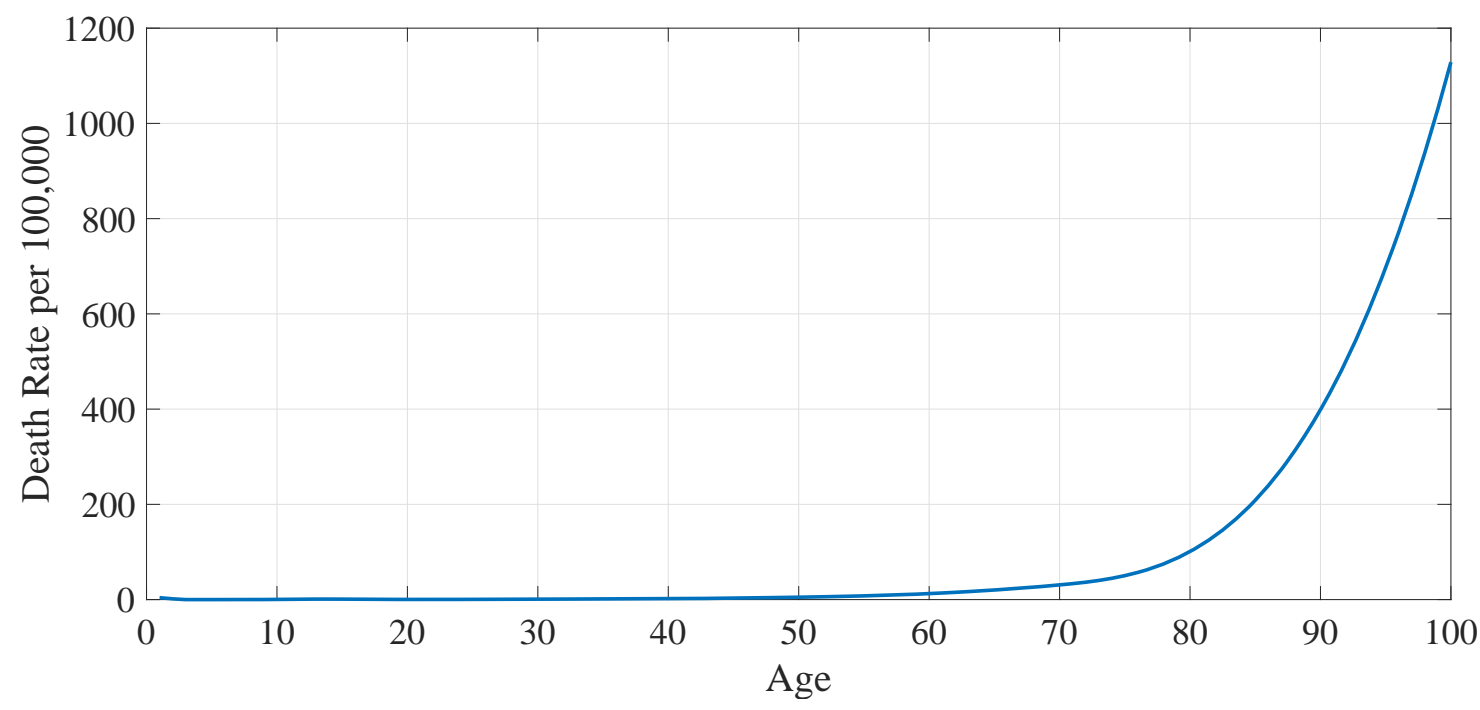

\begin{tabular}{lccccccccc}
\multicolumn{9}{c}{ FIGURE IV } \\
Death & Rate & Influenza & and & Pneumonia. & The graph & plots & the & in- \\
fluenza & and & pneumonia & death & rate per 100,000 & people in 2017 & by age.
\end{tabular}

By multiplying the death rate with the number of people $N_{i}$ of each age, we arrive at an

\footnotetext{
${ }^{7}$ See Table 6 of Centers for Disease Control and Prevention (2019b).
} 
estimated total number of deaths $D_{i}^{f l u}$ by age, which is plotted in Figure $\mathrm{V}$. The graph shows that the largest number of flu deaths happen at age 89 . The median age of flu deaths is approximately 85 years.

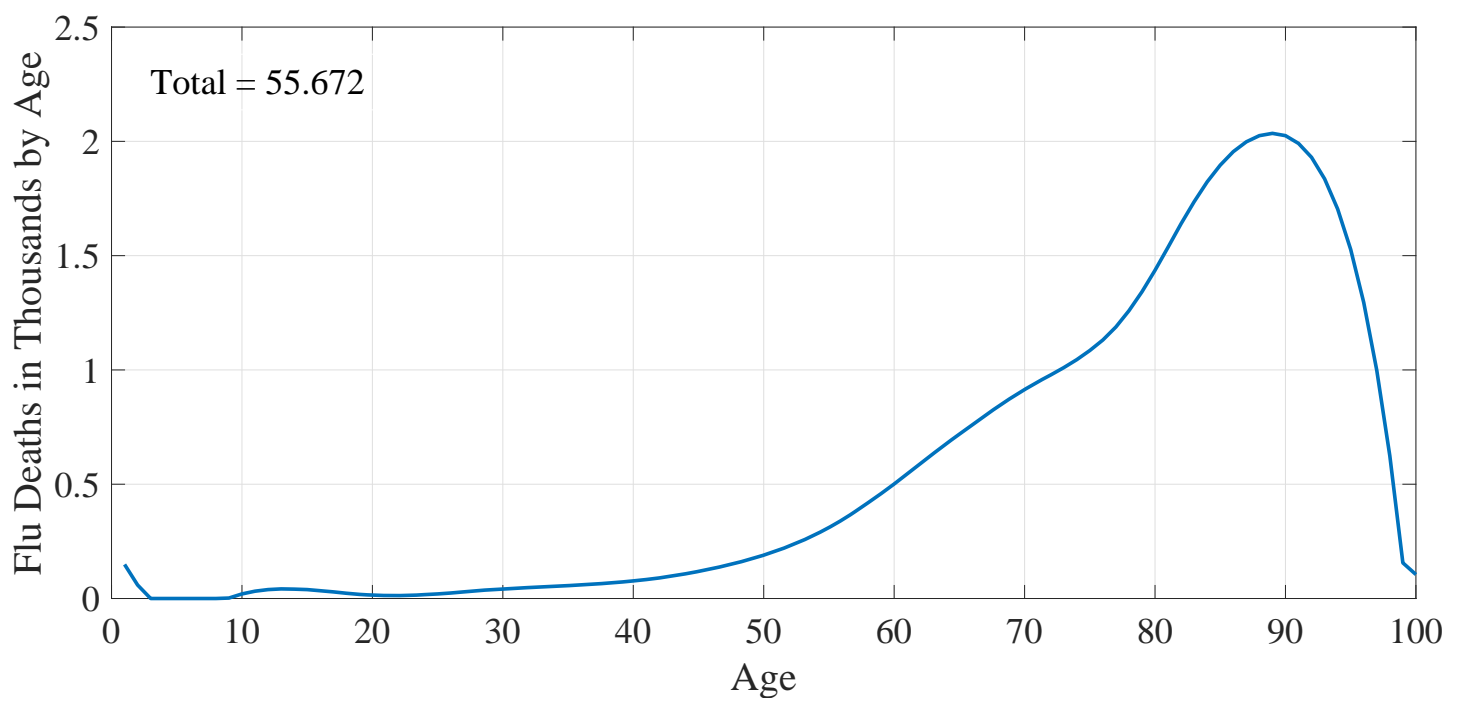

FIGURE V

Flu Deaths. The graph plots the total number of flu and pneumonia deaths in 2017 by age.

To compute the number of life years lost by age $\left(\Theta_{i}^{k}\right)$, we multiply the number of influenza deaths for each age by the average life expectancy at that age $]^{8}$

The results are plotted in Figure VI. The graph shows that accounting for life expectancy markedly changes the picture relative to the raw numbers of fatalities that was plotted in Figure $\mathrm{V}$. Babies that die from influenza lose approximately 80 life years. As a consequence, the number of life years lost is quite large for this age cohort, given that the death rate per 100,000 is 4 , compared to smaller numbers for older children and young adults.

\footnotetext{
${ }^{8}$ One concern related to using the average life expectancy at a given age is that agents dying from influenza may have a tendency to have had more severe pre-existing conditions than the rest of the population at that age, and thus, may typically have had a shorter life expectancy than the average. Yet, a similar selection effect applies for most other causes of deaths as well, in particular for COVID-19. As a result, this selection effect likely has minor implications for our estimates of the relative productivities across causes of deaths.
} 


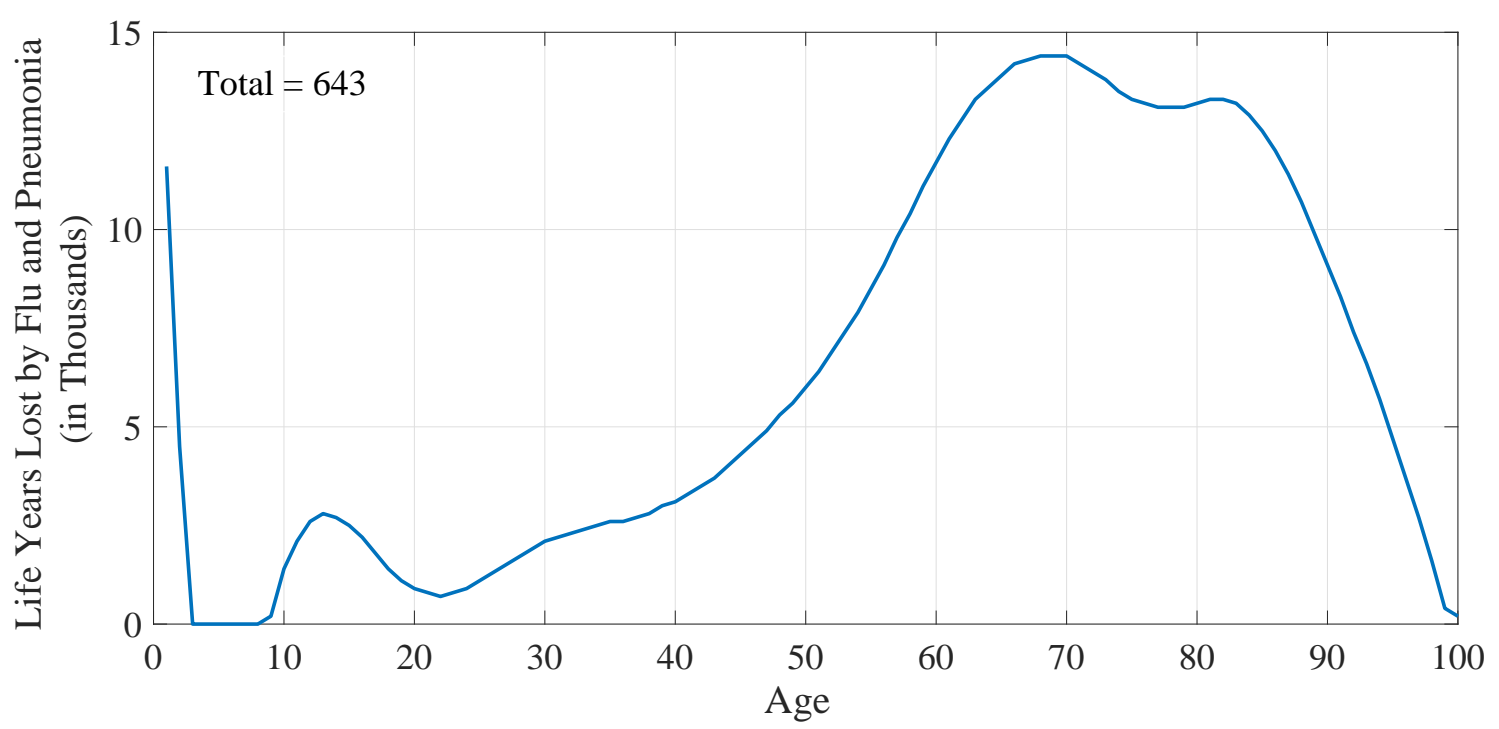

FIGURE VI

Life Years Lost Flu. The graph plots the life years lost due to influenza and pneumonia in 2017 by age.

\subsection{Vaccination Rates, Vaccine Effectiveness and Counterfactual Influenza Incidence}

The CDC reports influenza vaccination rates by age group. As before, we fit a spline through these data points to obtain an estimate of the vaccination rate by age cohort. The results are summarized in Figure VII The graph shows that in the lowest and highest age group, influenza vaccination exceeds $50 \%$, whereas for the majority of adults the rate is well below $50 \%$. Given the large number of life years lost due to influenza, it may be puzzling how low the vaccination rate is. Perhaps most surprisingly, the $\mathrm{CDC}$ reports that the vaccination rate among high-risk patients, that is, patients for which influenza could have substantial adverse effects due to existing medical conditions, is only a few percentage points higher than that for the low risk population? These data suggest limited selection effects related to underlying conditions in agents' current vaccination choices.

One last important input for our computations are estimates of the influenza vaccination effectiveness provided by the CDC. Influenza effectiveness is defined as follows ${ }^{10} \operatorname{Let} \operatorname{Pr}(\mathrm{Flu} \mid \mathrm{Vac})$ be the incidence of the flu among vaccinated patients, and let $\operatorname{Pr}(\mathrm{Flu} \mid \mathrm{NonVac})$ be the incidence

\footnotetext{
${ }^{9}$ See Centers for Disease Control and Prevention (2019a).

${ }^{10}$ The CDC states on its website: "The U.S. Flu VE Network currently consists of five study sites across the United States that measure the flu vaccine's effectiveness at preventing outpatient medical visits due to laboratoryconfirmed influenza." See Centers for Disease Control and Prevention (2020a).
} 


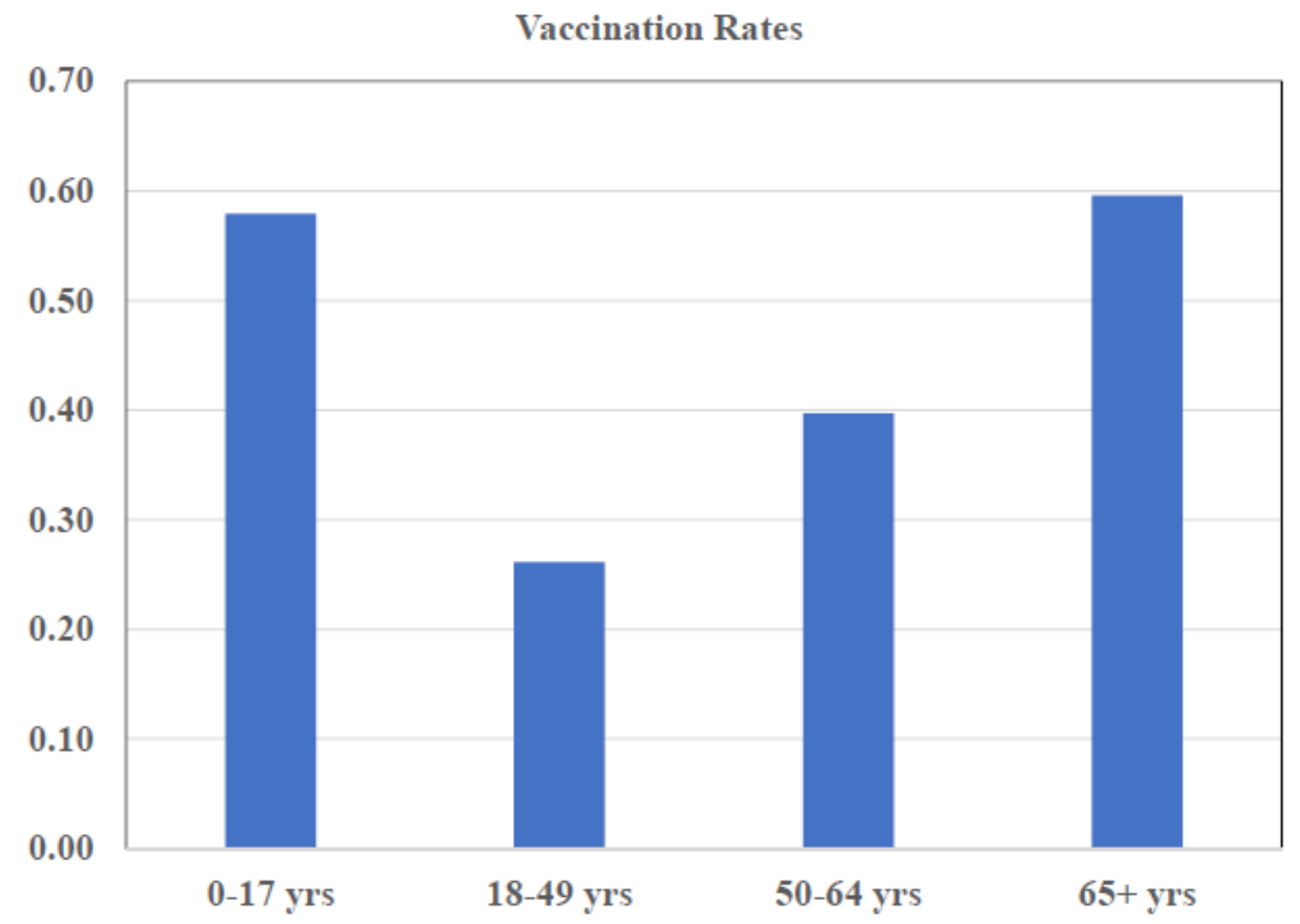

FIGURE VII

Vaccination Rates by Age Group. The bar graph plots the estimated fraction of vaccinated people by age group for the 2017-2018 flu season.

among non-vaccinated patients. Then the vaccine effectiveness (VE) is measured as:

$$
\mathrm{VE}=1-\frac{\operatorname{Pr}(\text { Flu } \mid \text { Vac })}{\operatorname{Pr}(\text { Flu } \mid \text { NonVac })}
$$

This definition implies that:

$$
\operatorname{Pr}(\text { Flu } \mid \text { Vac })=(1-\text { VE }) \operatorname{Pr}(\text { Flu } \mid \text { NonVac })
$$

In Figure VIII, we plot this effectiveness over the past 10 flu seasons. The vaccine effectiveness varies between $19 \%$ and $60 \%$ and has been fluctuating around $40 \%$, also in the most recent couple of flu seasons. The average over the 10 seasons is $43.8 \%$, with a $95 \%$ confidence bound of $[34.8,52.9]$.

Using these effectiveness numbers, we can compute a counterfactual influenza incidence as 


\section{Flu Effectiveness}

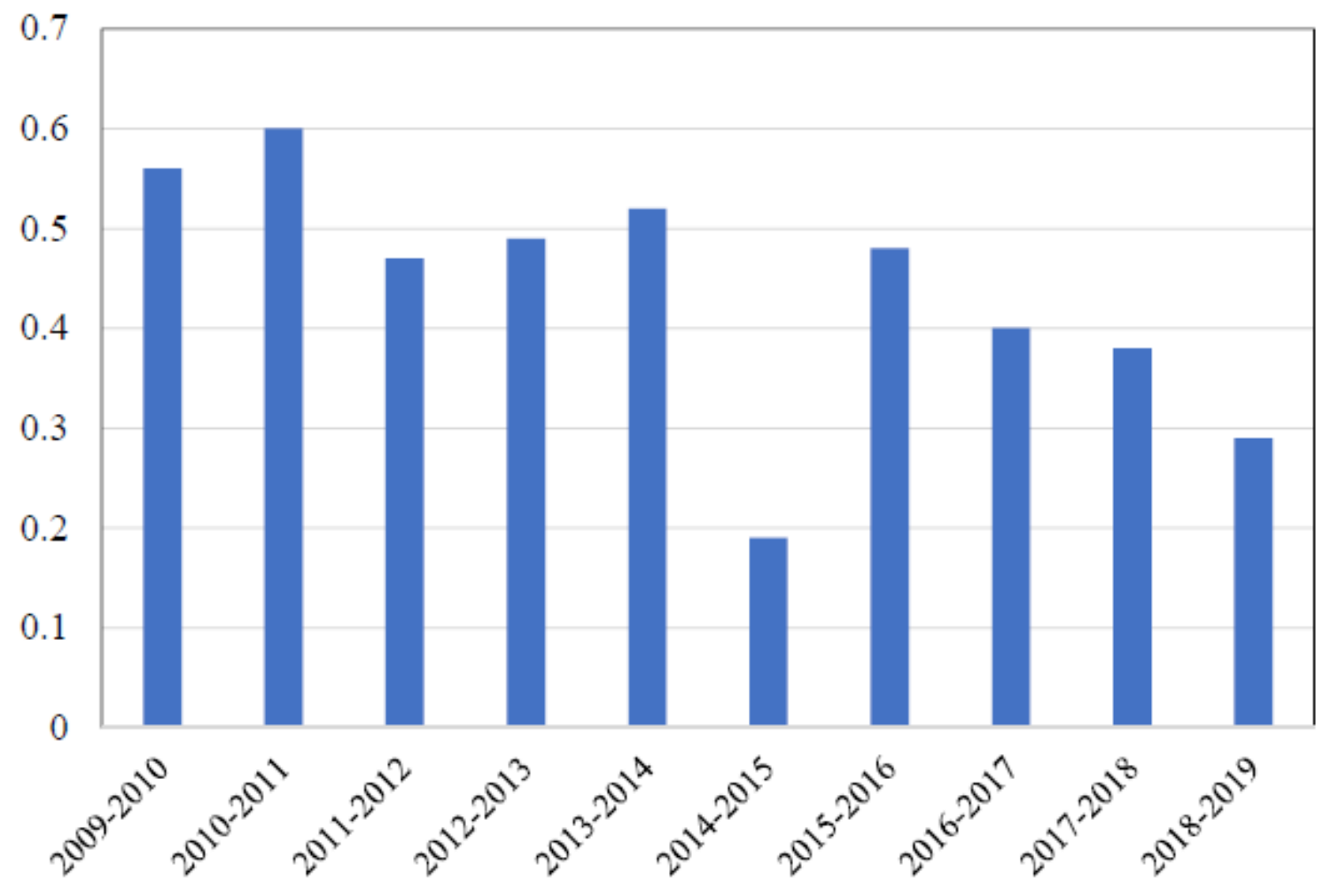

\section{FIGURE VIII}

Flu Vaccine Effectiveness by Flu Season. The bar graph plots the estimated flu vaccine effectiveness by flu season.

follows. First, we compute the probability with which agents in each age group experience a flu illness. We determine those numbers based on the 2017-2018 influenza season, by dividing the CDC's estimate of flu illnesses in the 2017-2018 flu season in each age group by the number of people in that age group (using the data reported in Figure \). The resulting estimates, denoted by $\operatorname{Pr}(\mathrm{Flu})$, are represented by the black solid line in Figure IX.

Next, we use the fact that the unconditional incidence of the flu in a given age cohort is a weighted average of incidences across the subgroups getting vaccinated and not vaccinated, respectively:

$$
\operatorname{Pr}(\text { Flu })=\operatorname{Pr}(\text { Flu } \mid \text { Vac }) \operatorname{Pr}(\text { Vac })+\operatorname{Pr}(\text { Flu } \mid \text { NonVac })(1-\operatorname{Pr}(\text { Vac })) .
$$


Combining this relation with Equation (5) yields

$$
\operatorname{Pr}(\text { Flu })=(1-\text { VE }) \operatorname{Pr}(\text { Flu } \mid \text { NonVac }) \operatorname{Pr}(\text { Vac })+\operatorname{Pr}(\text { Flu } \mid \text { NonVac })(1-\operatorname{Pr}(\text { Vac }))
$$

implying that

$$
\begin{aligned}
\operatorname{Pr}(\text { Flu } \mid \text { NonVac }) & =\frac{\operatorname{Pr}(\text { Flu })}{1-\mathrm{VE} \times \operatorname{Pr}(\text { Vac })} \\
\operatorname{Pr}(\text { Flu } \mid \text { Vac }) & =(1-\text { VE }) \operatorname{Pr}(\text { Flu } \mid \text { NonVac }) .
\end{aligned}
$$

In Figure IX, we plot the conditional flu incidences for both vaccinated and non-vaccinated agents. Using these incidence rates, we can also estimate the counterfactual total life years lost

\section{Flu Incidence}

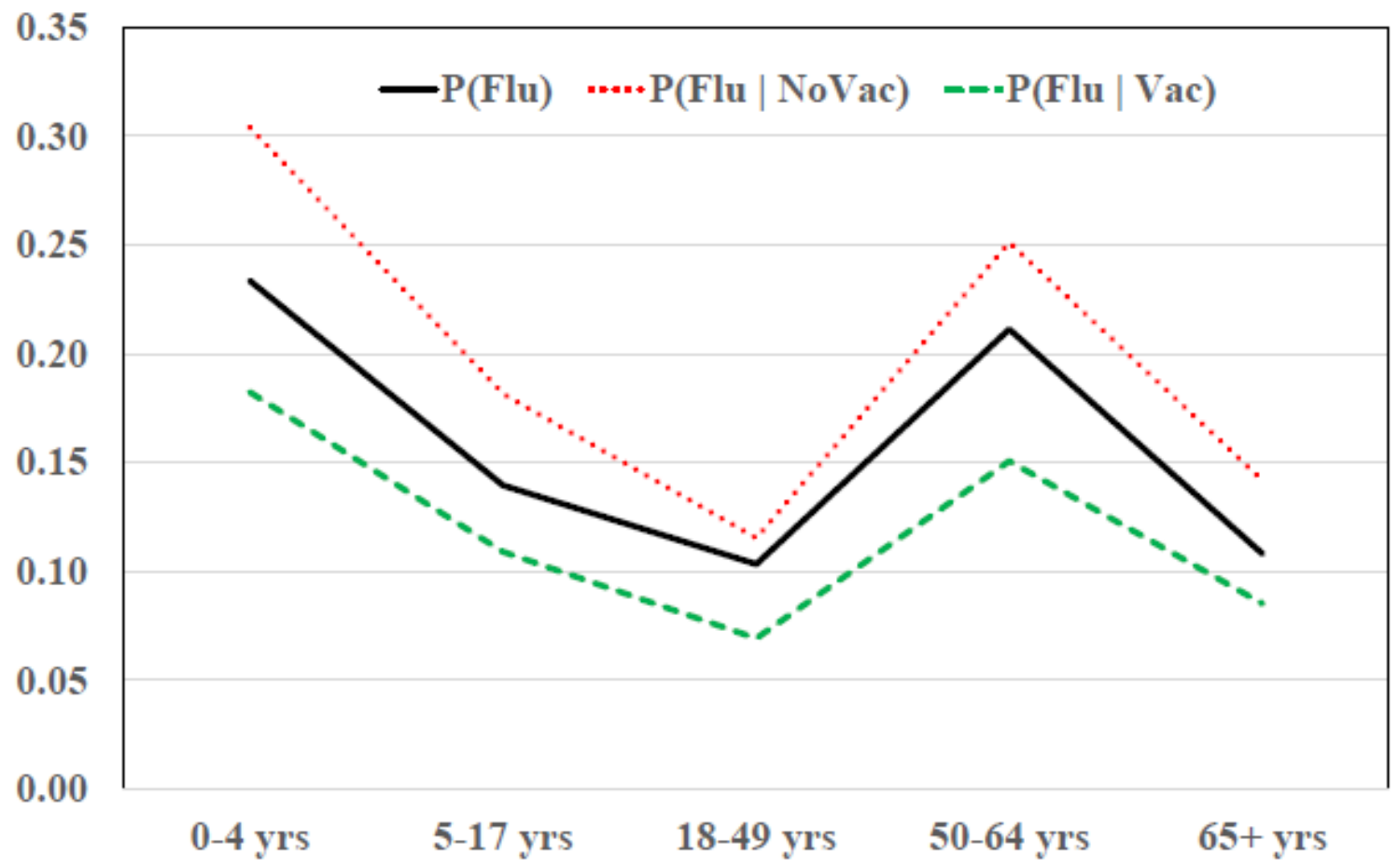

FIGURE IX

Influenza Incidence by Vaccination Status and Age Group. The graph plots the flu incidence by age group in the 2017-2018 flu season. The three lines represent the incidence among the entire age group (in black), as well as among those individuals within each age group that were vaccinated (in green) and not vaccinated (in red), respectively.

due to influenza, in the scenario where the whole population is vaccinated. Note that the actual 
flu death rate for each age can be written as:

$$
r_{i}^{\text {flu }}=\operatorname{Pr}(\text { Death } \mid \text { Flu }) \times \operatorname{Pr}(\text { Flu }) \times 100,000 .
$$

We can now compute the counterfactual death rate as:

$$
r_{i}^{\text {flu-cf }}=\operatorname{Pr}(\text { Death } \mid \text { Flu }) \times \operatorname{Pr}(\text { Flu } \mid \text { Vac }) \times 100,000 .
$$

As a baseline scenario, we use a flu vaccine effectiveness estimate of 0.4 , which is lower than the average flu effectiveness of 0.438 over the past 10 years, and close to the flu effectiveness estimates for the 2017-2018 season. While there could exist a different vaccine effectiveness by age group, there seems to be no scientific consensus on the existence of such differences and their direction ${ }^{11}$ As a result, we assume that the effectiveness is uniform across age cohorts.

We then proceed with our calculations in the same manner as before and recompute the life years lost from the flu. Figure $\mathrm{X}$ compares the life years lost by age for the counterfactual scenario to those of the actual data (as was summarized in Figure VI). The graph shows that in

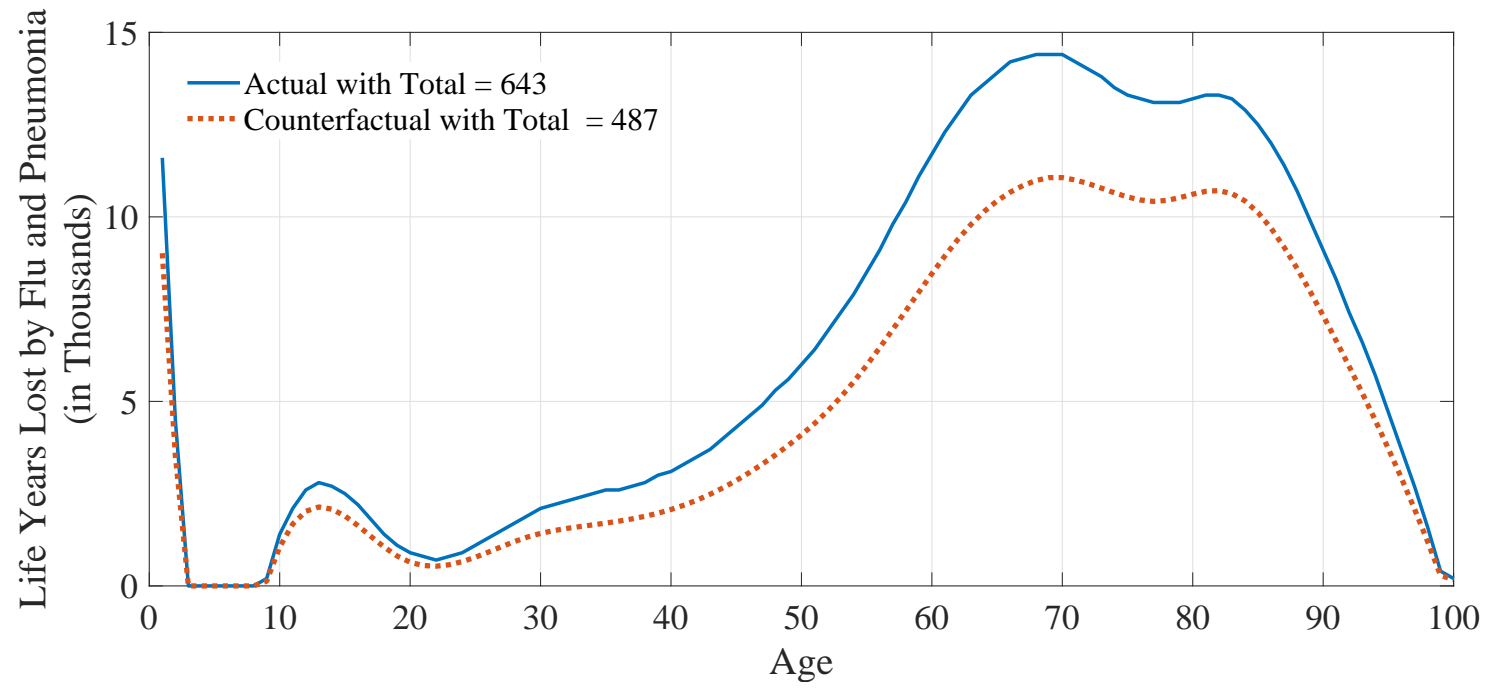

FIGURE X

Life Years Lost Flu and Pneumonia. The graph plots the life years lost due to the flu by age as well as the counterfactual scenario of full vaccination. The data is calibrated to a total number of flu deaths of 55,672 and uses death rates from 2017.

this case an estimated 156,000 could have been saved by making vaccination mandatory. There are substantial gains both for very young children (because of their long life expectancy) as

\footnotetext{
${ }^{11}$ See Russell et al. (2018).
} 
well as for the population with ages between 60 and 70 years. For the group of 65 -year olds, the savings are the largest, at 3,500 life years.

These computations are conservative in the following sense. If the entire population were vaccinated, influenza related fatalities could likely be further reduced due to positive externalities that are not incorporated into our calculations underling Figure $\mathrm{X}$. In particular, once a critical mass of agents in a population becomes immune due to vaccination, a virus cannot spread as effectively, as "herd immunity" operates. This type of externality protects both those agents that are not getting vaccinated and those that do not achieve immunity by getting vaccinated themselves. In the best case scenario, a virus completely ceases to spread. If mandatory vaccination achieved full herd immunity, approximately 643,000 life years could be saved in a year like 2017.

How about other flu-seasons? In Figure $\mathrm{XI}$ we plot the flu deaths by season for the past decade as estimated by the CDC. These calculations are part of the CDC's flu burden calculations. The graph shows that the 2017-2018 flu season was particularly severe. Flu deaths range between 12 and 61 thousand deaths per year, with an average of 37,460 deaths per year.

Holding the cross-sectional distribution of deaths across ages fixed and applying the life expectancy numbers of 2017 , this implies an average number of life years lost equal to:

$$
\frac{37,460}{55,672} \times 642,935=432,655
$$

and a number of life years saved through mandatory vaccination equal to:

$$
\frac{37,460}{55,672} \times 156,300=105,170
$$

\subsection{Effectiveness of Investments in Influenza Mitigation}

In this section, we determine the effectiveness of investments in influenza mitigation. As we have computed in the previous sections, an estimated 156,300 life years could have been saved in 2017 by vaccinating the entire U.S. population. For the average flu season we have estimated this number to be 105,170 .

How expensive would be such a policy of mandatory vaccination? The CDC publishes a list of the cost of all vaccines. It reports that flu vaccinations vary in CDC cost per dose between $\$ 11.67$ and $\$ 18.19$. For private sector costs per dose, the numbers vary between $\$ 16.93$ 
Flu Deaths by Season

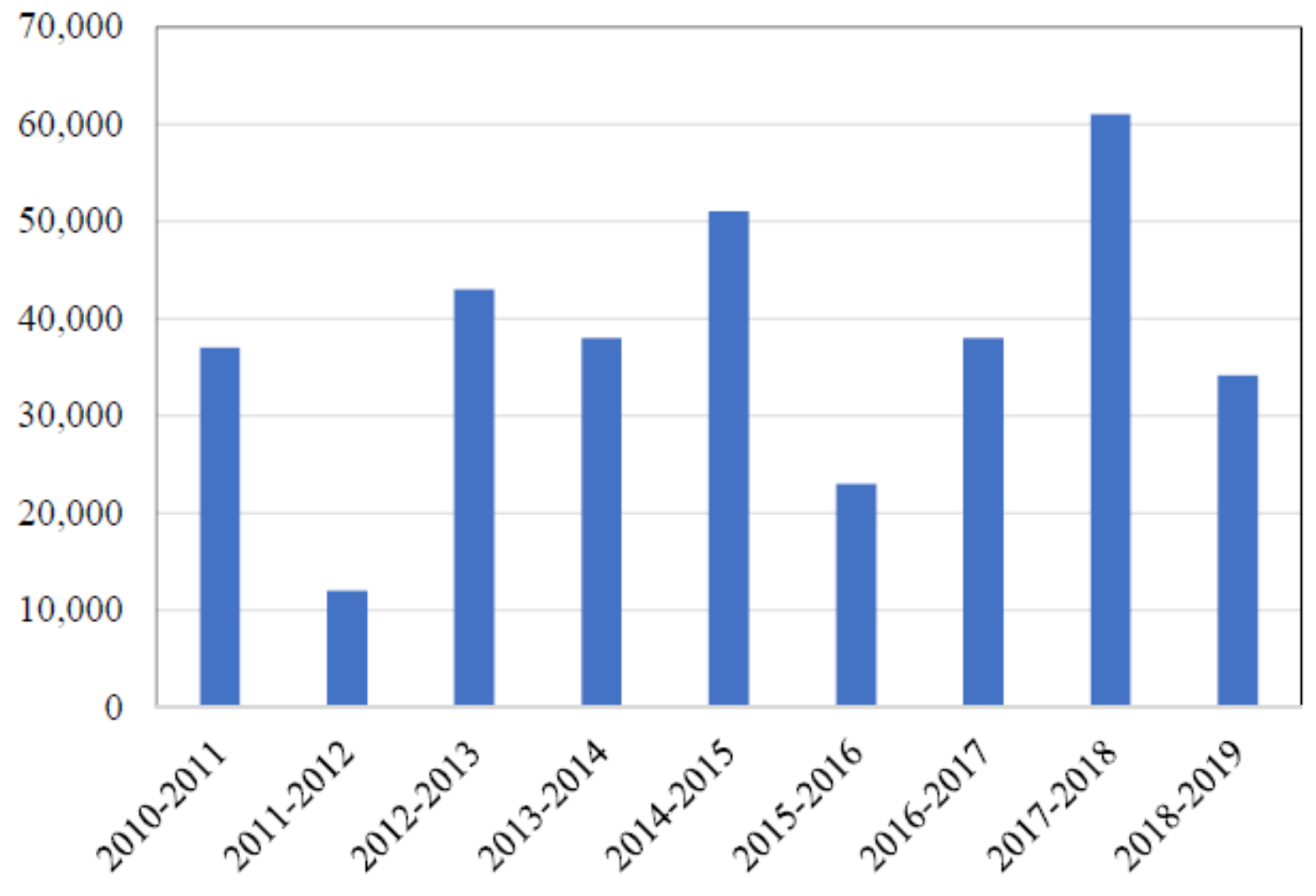

FIGURE XI

Flu Deaths by Season. The graph plots the number of influenza deaths by season.

and $\$ 32.47 \sqrt[12]{12}$ Based on these numbers, and to be conservative, we will work in our baseline computations with a price of $\$ 20$ per dose. Given that an estimated total of 192.5 million people are not vaccinated, the cost to vaccinate all of them equals $\$ 3.85$ Billion.

For the calendar year 2017 this implies the following costs of gaining one life year through influenza mitigation:

Cost per Life Year Gained From Influenza Mitigation $=\frac{\$ 3.85 \text { Billion }}{156,300}=\$ 24,600$.

For an average flu year, this number equals:

Cost per Life Year Gained From Influenza Mitigation $=\frac{\$ 3.85 \text { Billion }}{105,170}=\$ 36,607$.

For the remainder of our computations, we will work with this latter number.

\footnotetext{
${ }^{12}$ See Centers for Disease Control and Prevention (2020b).
} 


\section{COVID-19}

In this section, we study the effectiveness of COVID-19 mitigation investments. Whereas our objective is to shed light on the marginal effectiveness of such investments, we compute an average effectiveness for identification purposes. Due to the general presence of decreasing returns to scale, this average effectiveness can be used as an upper bound estimate for the marginal measure. To compute an average value, we compare realized and projected outcomes under the status quo mitigation policies to those under a counterfactual no mitigation policy. This no mitigation policy is not per se a desirable scenario, but it provides a benchmark that can be associated with plausible projections for outcomes.

Under this no mitigation scenario, a large fraction of the population would become infected in a short period of time. Greenstone and Nigam (2020) estimate that in such a scenario 1.7 million Americans would die from COVID-19 by July 2020, at which point the pandemic would end due to herd immunity. A substantial fraction of these deaths would result from overextended hospitals and lack of medical care. In Report 9 of Ferguson et al. (2020), the authors estimate that $81 \%$ of patients would get infected before herd immunity kicks in. They further estimate that 2.2 million people would die in “... the (unlikely) absence of any control measures or spontaneous changes in individual behaviour." For our computations, we will work with these latter numbers. Given an estimated death toll of 2.2 million people, combined with an infection rate of $81 \%$, this number implies an infection fatality rate (IFR) of $0.831 \%$. The incidence rates (probabilities) are then given by:

$$
\begin{aligned}
\operatorname{Pr}(\text { COVID-19 }) & =0.81 \\
\operatorname{Pr}(\text { Death } \mid \text { COVID-19 }) & =0.00831 .
\end{aligned}
$$

The unconditional probability of dying from COVID-19 in that case is the product of those two probabilities, and equal to $0.66 \%$.

Next, we use CDC data to calibrate infection fatality rates by age group, and, as before, fit a spline through the available cohort data points and rescale estimates so that they are consistent with the overall IFR stated above. The resulting age-specific infection fatality rates are plotted in Figure XII. Multiplying these age-specific rates by 0.81 results in the projected COVID-19 death rate by age in 2020, which is plotted in Figure XIII.

Based on these death rates we compute the estimated life years lost from COVID-19 assuming no mitigation. The results are plotted in Figure XIV. The total number of life years lost in 


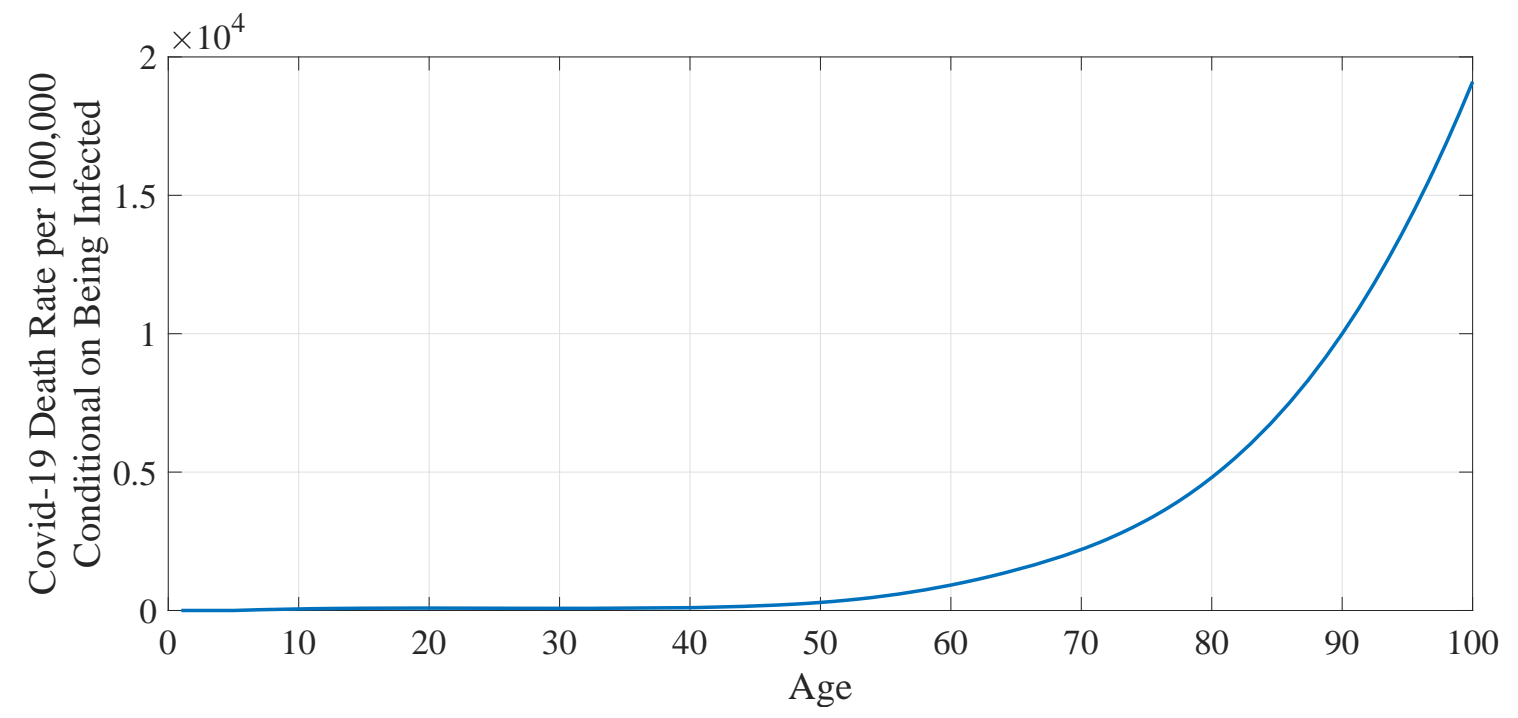

FIGURE XII

COVID-19 Infection Fatality Rate by Age. The graph plots the infection fatality rate (IFR) for COVID-19 by age.

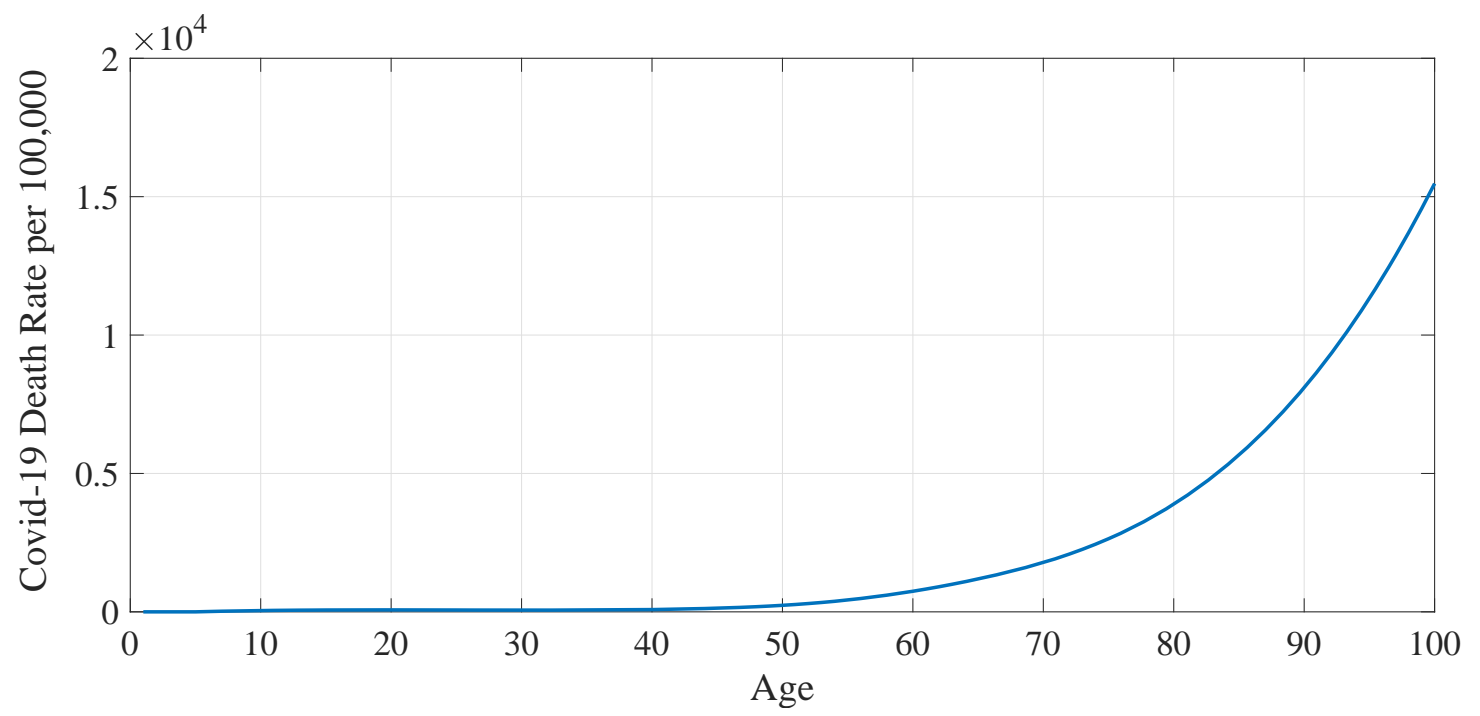

FIGURE XIII

COVID-19 Death Rate by Age. The graph plots the COVID-19 death rate by age under a scenario of no mitigation, assuming that $81 \%$ of the population will become infected.

this scenario is 33.1 million. To determine the number of life years gained through mitigation, we subtract from this number the life years lost from COVID-19 under the current mitigation policies. At the time of this paper's writing, forecasts predict 130,000 deaths this year, which corresponds to approximately 1.956 million life years. In sum, we obtain 31,152 million life years gained through mitigation. 


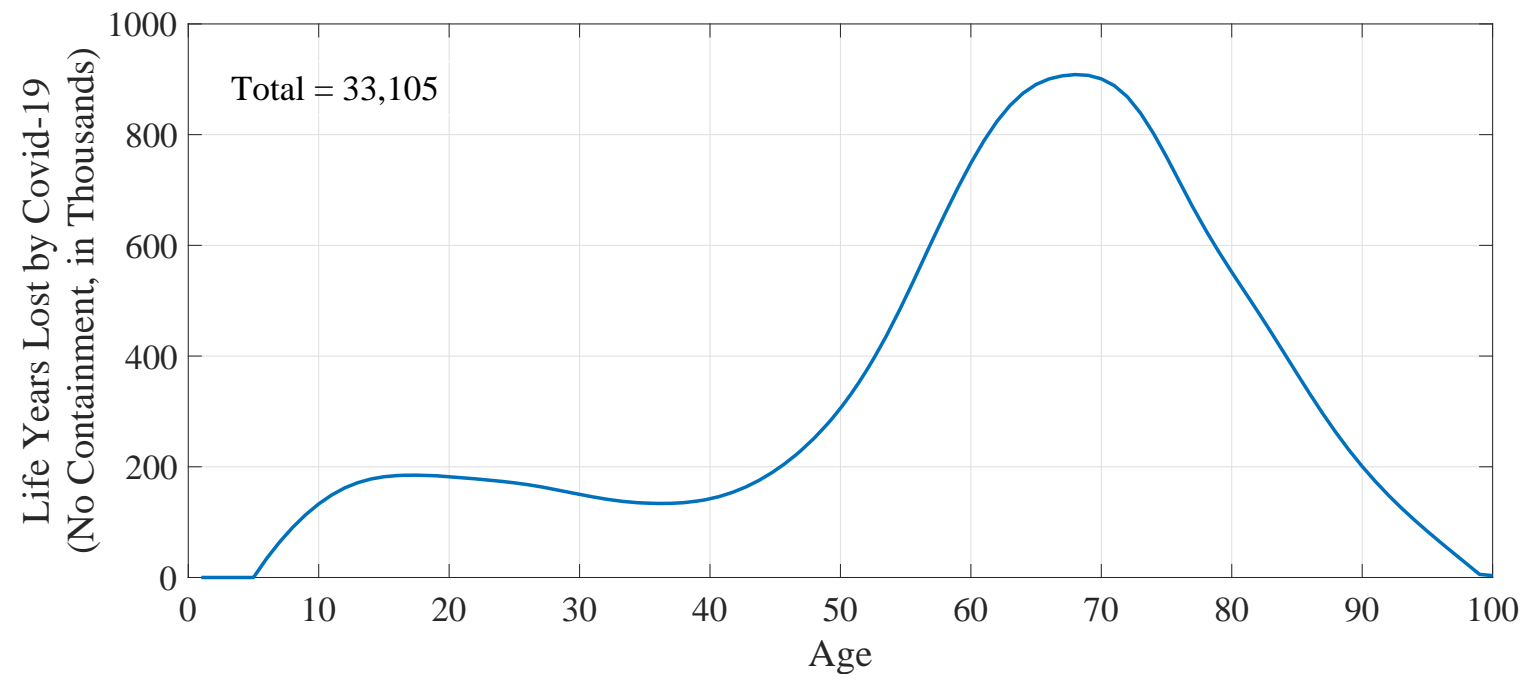

COVID-19 Life Years Lost by Age. $\quad$ The graph plots the esti-

Next, we estimate GDP growth in the no mitigation scenario, in which case the pandemic is predicted to end in July 2020. Existing studies suggest that about $97.4 \%$ of agents getting infected with COVID-19 (which, in turn, represent about $81 \%$ of the population) have mild symptoms, in the sense that they do not require hospitalization. ${ }^{13}$ In addition, $19 \%$ of agents do not get infected. To incorporate the associated implications for economic activity and given real GDP growth forecasts of about $2 \%$ prior to the onset of the pandemic, we assume $0 \%$ growth in 2020 in the no mitigation scenario, implying a loss of $2 \%$ of GDP due to COVID-19 disruptions. In contrast, under the current mitigation policies, the projections for GDP growth are approximately $-6 \%$ for $2020 .{ }^{14}$ Taking the difference between these two GDP growth estimates and multiplying with the GDP in 2019, we obtain an implied mitigation investment of about $\$ 1.3$ trillion.

This estimate is conservative in that it only accounts for losses reflected in this year's GDP (a flow), although this year's shocks to the stocks of human capital (e.g., due to unemployment) and physical capital (e.g., due to bankruptcies) are likely to lower GDP also in the next 1 to 2 years. It also is worth noting that this estimate is significantly below the $\$ 2$ trillion costs of the three coronavirus-relief packages enacted by the Trump administration and Congress.

\footnotetext{
${ }^{13}$ See Salje et al. (2020).

${ }^{14}$ See, e.g., International Monetary Fund (2020).
} 
Combining these numbers, we obtain the following effectiveness estimate for COVID-19:

Cost per Life Year Gained From COVID-19 Mitigation $=\frac{\$ 1.3 \text { Trillion }}{31.152 \text { Million }}=\$ 41,730$.

This cost estimate is somewhat higher than the cost of a life year gained from influenza mitigation (see equation (11)).

Alternatively, we can compute the implied COVID-19 mitigation value by multiplying the estimated number of life years lost by COVID-19 with the cost per life year gained from influenza mitigation, which we have estimated to be $\$ 36,607$ :

Implied COVID-19 Mitigation Value $=31.152$ Million $\times \$ 36,607=\$ 1.14$ Trillion.

\section{Sensitivity Analysis and Discussion}

In this section we explore the sensitivity of our results with respect to several key assumptions. Due to data limitations, we are, however, not conducting sensitivity analyses for the following assumptions:

1. Counterfactual life expectancies of people dying of the flu or of COVID-19 are equal to the age-specific average life expectancies in the population.

2. We do not distinguish between men and women, despite the fact that men die more often from COVID-19 and have a shorter life expectancy.

Throughout, we have been conservative in our baseline assumptions, in the sense that they consistently lower the estimated dispersion between the effectiveness of influenza and COVID19 mitigation. Assumption 2 is, in that sense, also conservative.

Assumption 1 has a somewhat more ambiguous effect. The vast majority of COVID-19 patients have underlying health conditions that likely make their life expectancy shorter than the average life expectancy in a given age cohort. Yet, this selection effect likely also applies for influenza. Whether this selection effect is stronger for COVID-19 than for influenza is difficult to assess at this point. It is, however, plausible that the two effects roughly cancel, when it comes to the difference between the effectiveness estimates ((11)) vs. (14)).

Next, we will conduct explicit sensitivity analyses for the following assumptions: 
1. The price of the influenza vaccine equals $\$ 20$ per dose.

2. Influenza vaccine effectiveness equals $40 \%$.

3. Even if $100 \%$ of the U.S. population are vaccinated, positive externalities associated with herd immunity do not apply.

4. Herd immunity for COVID-19 is achieved at $81 \%$.

5. The COVID-19 infection fatality rate is $0.831 \%$.

6. All computations are on a one-year basis.

In Table 1 we report the sensitivity of our results with respect to these assumptions by using informed ranges for the parameters. We report cost per life year gained from influenza mitigation (see equation (11)) and the corresponding implied COVID-19 mitigation value (see equation (15)).

Given that we have already assumed in our baseline analysis that the remaining 192.5 million Americans that are currently not vaccinated will be vaccinated, implying an investment of $\$ 3.85$ Billion, we cannot choose a higher level. Yet, we also consider the scenario where reaching a vaccination level of $70 \%$ would be sufficient to achieve the same gains in life years as estimated in our baseline numbers, due to the effects of herd immunity (the "low" scenario). In this case, only an additional 94.4 million Americans have to be vaccinated, with the same effect on life years gained.

The table shows that our results are most sensitive to the flu effectiveness assumption and the death rate assumption. If the flu vaccine is close to ineffective (in the $20 \%$ range) or the death rate surpasses $1.4 \%$, the estimated COVID-19 containment value surpasses the $\$ 1$ Trillion mark.

\section{Life Years Lost from Other Diseases}

In this last section, we discuss other major causes of death. Table 2 lists the total number of (estimated) deaths for each disease, the total number of life years lost, the average of the number of life years lost per death (i.e. the ratio of the life years lost and the total number of deaths), as well as the mitigation value implied by our estimate for influenza vaccine investments' effectiveness (see equation (15)). The latter is computed by multiplying the life years lost by $\$ 24,600$. 
The table illustrates that fewer life years per death are lost to flu than to COVID-19. As we have argued before, this puts into context the statement that COVID-19 particularly affects older people, as it does less so than the flu. The same is true for heart disease which is the most common cause of death in the U.S. (not reported) and Alzheimer's disease (not reported). 


\begin{tabular}{|c|c|c|c|c|c|c|c|c|c|}
\hline \multirow[b]{2}{*}{ Parameter } & \multicolumn{3}{|c|}{ Parameter Range } & \multicolumn{3}{|c|}{ Cost per Life Year (Flu) } & \multicolumn{3}{|c|}{$\begin{array}{c}\text { Implied COVID-19 } \\
\text { Mitigation Value (Billion) }\end{array}$} \\
\hline & Low & Baseline & High & Low & Baseline & High & Low & Baseline & High \\
\hline Covid-19 Average Death Rate & 0.0030 & 0.00831 & 0.015 & $\$ 36,608$ & $\$ 36,608$ & $\$ 36,608$ & $\$ 540.2$ & $\$ 1,140.4$ & $\$ 2,116.1$ \\
\hline Flu Vaccine Effectiveness & $60 \%$ & $40 \%$ & $20 \%$ & $\$ 20,877$ & $\$ 36,608$ & $\$ 79,538$ & $\$ 650.4$ & $\$ 1,140.4$ & $\$ 2,477.8$ \\
\hline Vaccine Price & $\$ 12$ & $\$ 20$ & $\$ 28$ & $\$ 21,965$ & $\$ 36,608$ & $\$ 51,251$ & $\$ 684.2$ & $\$ 1,140.4$ & $\$ 1,596.6$ \\
\hline Covid-19 Herd Immunity & $60 \%$ & $81 \%$ & $100 \%$ & $\$ 36,608$ & $\$ 36,608$ & $\$ 36,608$ & $\$ 826.2$ & $\$ 1,140.4$ & $\$ 1,424.7$ \\
\hline Flu Immunization Level Required & $70 \%$ & $100 \%$ & - & $\$ 17,952$ & $\$ 36,608$ & - & $\$ 559.2$ & $\$ 1,140.4$ & - \\
\hline
\end{tabular}

\section{Table 1}

Sensitivity Analysis. The Table reports sensitivity analyses for a number of parameters. Parameters are ordered in terms of sensitivity importance. Ranges are defined "low" and "high" based on their influence on the outcome variables: Cost per Life Year (Flu) and Implied COVID-19 Mitigation Value. For all parameters lower (higher) values lead to lower (higher) values of the outcome variables, with the exception of the flu vaccine effectiveness (VE) rate. 


\begin{tabular}{l|cccr}
\hline Unit & $\begin{array}{c}\text { Total Deaths } \\
\text { People (Thousands) }\end{array}$ & $\begin{array}{c}\text { Life Years Lost } \\
\text { Years (Thousands) }\end{array}$ & $\begin{array}{c}\text { Life Years Lost per Death } \\
\text { Years }\end{array}$ & $\begin{array}{r}\text { Flu-Implied Mitigation Value } \\
\$ \text { Billion }\end{array}$ \\
\hline COVID-19 (No containment) & 2,200 & 33,108 & 15.0 & $\$ 1,212$ \\
COVID-19 (Containment) & 130 & 1,956 & 15.0 & $\$ 72$ \\
COVID-19 (Difference) & 2,070 & 33,108 & 15.0 & $\$ 1,140$ \\
& & & & 11.7 \\
Flu & 55 & 643 & 15.6 & $\$ 24$ \\
Cancer & 599 & 9,370 & 36.4 & $\$ 55$ \\
Traffic Deaths & 41 & 1,497 & 35.2 & $\$ 61$ \\
Suicides & 47 & 1,661 & 34.6 & $\$ 139$ \\
Alcohol and Drugs & 110 & 3,798 & & \\
\hline
\end{tabular}

Other causes of death. Total deaths, life years lost, and mitigation values implied by influenza vaccine investments' effectiveness for several common causes of death 


\section{Long-term Valuations}

Our computations have focused on the costs and benefits for one year. Given that many of the preventive life preserving investments are annual investments, this gives a first impression of the tradeoffs that we study. However, in reality there are several reasons why multi-period computations are useful, implying that the present value of costs and benefits becomes the object of interest. For example, suppose that vaccinating the entire population achieves herd immunity and could largely eliminate one of the flu strains, then this one-time investment could save life years over many years to come. Similarly, investing in cancer research affects the stock of knowledge available in all future years, implying perpetual savings of life years. The numbers in Table 2 would be substantially larger once such long-term effects are taken into account.

At the time of the writing of this paper, there is still substantial debate about whether there will be several waves of COVID-19 infections in the coming years. A large fraction of the mitigation measures currently implemented (e.g., lockdowns and social distancing) are not targeting the elimination of the virus, but rather intend to delay its spread. That is, these types of measures undertaken in 2020 would not create incremental benefits in future years, relative to the benefits we accounted for in our calculation for 2020 (the savings of 31.152 Million life years).

\section{Conclusion}

In this paper, we analyzed the relative effectiveness of investments aimed at increasing agents' life expectancy, with a particular focus on influenza and COVID-19 mitigation. Based on estimates for the marginal effectiveness of influenza vaccines, we shed light on the level of COVID-19 mitigation investments that would imply an approximate equalization of influenza and COVID-19 mitigation activities' expected effectiveness. Given current projections for COVID-19 mitigation costs, our results suggest that wide-spread influenza vaccination would be a highly effective life-preserving investment.

\section{References}

Centers for Disease Control and Prevention (2019a): "Flu Vaccination Coverage, United States, 2016-17 Influenza Season," Website: https : / /www • cdc.gov/flu/ 
fluvaxview/coverage-1617estimates.htm.

(2019b): “National Vital Statistics Reports - Deaths: Final Data for 2017," Website: https://www.cdc.gov/nchs/data/nvsr/nvsr68/nvsr68_09-508.pdf.

(2020a): “CDC Seasonal Flu Vaccine Effectiveness Studies," Website: https:// WwW.cdc.gov/flu/vaccines-work/effectiveness-studies.htm

- (2020b): "CDC Vaccine Price List," Website: https://www.cdc.gov/ vaccines/programs/vfc/awardees/vaccine-management/price-list/ index.html.

FERGUSON ET AL. (2020): "Report 9: Impact of non-pharmaceutical interventions (NPIs) to reduce COVID-19 mortality and healthcare demand," https://www.imperial.ac.uk/media/imperial-college/medicine/sph/ide/gidafellowships/Imperial-College-COVID19-NPI-modelling-16-03-2020.pdf.

Greenstone, M., And V. Nigam (2020): “Does Social Distancing Matter?," Website: https://bfi.uchicago.edu/working-paper/2020-26/.

International Monetary Fund (2020): “United States and the IMF,” Website: https: //WWW.imf.org/en/Countries/USA.

RUSSELL ET AL. (2018): "Influenza vaccine effectiveness in older adults compared with younger adults over five seasons.," Vaccine, 36(10), 1272-1278.

SALJE ET AL. (2020): "Estimating the burden of SARS-CoV-2 in France," Website: https: //www.medrxiv.org/content/10.1101/2020.04.20.20072413v2.

World Health Organization (2020): "Q\&A: Influenza and COVID-19 - similarities and differences," Website: https://www.who.int/emergencies/diseases/ novel-coronavirus-2019/question-and-answers-hub/q-a-detail/ q-a-similarities-and-differences-covid-19-and-influenza. 